\title{
THERMOMECHANICALLY-CONSISTENT Phase-Field Modeling of Thin Film Flows
}

\author{
Christopher Miles ${ }^{* \dagger}$, Kristoffer G. van der Zee ${ }^{* \dagger}$, \\ Matthew E. Hubbard ${ }^{\dagger}$ and Roderick MacKenzie ${ }^{\ddagger}$
}

25 June 2018

\begin{abstract}
We use phase-field techniques coupled with a Coleman-Noll type procedure to derive a family of thermomechanically consistent models for predicting the evolution of a non-volatile thin liquid film on a flat substrate starting from mass conservation laws and the second law of thermodynamics, and provide constraints which must be met when modeling the dependent variables within a constitutive class to ensure dissipation of the free energy. We show that existing models derived using different techniques and starting points fit within this family. We regularise a classical model derived using asymptotic techniques to obtain a model which better handles film rupture, and perform numerical simulations in 2 and 3 dimensions using linear finite elements in space and a convex splitting method in time to investigate the evolution of a flat thin film undergoing rupture and dewetting on a flat solid substrate.
\end{abstract}

\section{Contents}

1 Introduction $\quad 2$

2 Axioms 3

3 Constitutive Dependence 4

*Corresponding authors, \{Christopher.Miles , KG.vanderZee\}@nottingham.ac.uk

${ }^{\dagger}$ School of Mathematical Sciences, University of Nottingham

${ }^{\ddagger}$ Faculty of Engineering, University of Nottingham 
5 Choices and Connections 5

6 Regularisation of the Asymptotic Model 6

7 Conclusion $\quad 8$

\section{Introduction}

The ability to accurately predict the evolution of the morphology of a thin film has a large range of applications, from the linings of mammalian lungs in biophysics and lava flows in geology [11] to the fabrication of thin-film solar cells [6]. In the last case, it is particularly key to know the final morphology of the film, as dewetting of the film on the substrate, driven by a combination of evaporation and interaction energies such as disjoining pressures can cause poor surface coverage, resulting in low device efficiency [3].

There are two main methods for developing a model to describe thin film evolution. The first method is an asymptotic approach which assumes density, viscosity and thermal conductivity are negligible in the vapour phase of the system, and employs a long-wave approximation where it is assumed that the gradients of the height and temperature functions are small in the area considered. This methodology is demonstrated in full complexity by Burelbach et al [1]. This method is rigorous in its derivation in the sense that the resulting thin-film equations are obtained from the bulk fluid equations. However, the resulting thin-film model may have difficulty handling film rupture, when the height of the film becomes zero at a point, and a hole forms.

The second method is an energy-gradient dynamics approach directly applied to the film height, in which it is postulated that the energy of the system dissipates according to gradient dynamics, and that the film grows towards an equilibrium which is achieved at the minimal energy. Thiele [15] uses this method to build on the work in [12] to describe phenomena such as dewetting and evaporation in thin films. While this model is less rigorously derived, it is able to naturally cope with film rupture.

In this paper, we introduce a general derivation for a family of thin-film flow models based on the classical theory of thermomechanics and the Coleman-Noll procedure $[7,2]$. We derive the family of models using as a starting point the fundamental axioms of conservation of mass and the second law of thermodynamics. Following [5], we stipulate that the free energy of the film $\Psi$ depends on the film's height and its gradient, $\Psi=\widehat{\Psi}(h, \nabla h)$. We then derive constraints on the 
remaining constitutive variables, and allow these to additionally depend on the variational derivative $\mu$ of the total free energy and its gradient, $\nabla \mu$. This ensures energy dissipation for allowed choices made by the modeler. We show that the above-mentioned models developed in [15] and [1] fit into our framework despite having been derived from different starting points and with different techniques. Finally, numerical simulations show how a small perturbation in a flat film evolves. In order to enable the more rigorously derived asymptotic model to progress past the point of film rupture, we use phase-field techniques to regularise this model within our framework, and thereby guarantee consistency with the second law of thermodynamics, i.e. free energy dissipation.

This paper is structured as follows. In Section 2 we derive a basic framework for thin film models. We follow phase-field arguments to derive a family of simple models to describe the evolution of thin films while ensuring energy dissipation in Sections 3 and 4. In Section 5, we show that the model derived in [1] fits into this family when evaporation is not taken into consideration. In Section 6, the model is regularised and discretised using linear finite elements and numerical experiments are carried out. Finally, concluding remarks are made in Section 7.

\section{Axioms}

There are two key principles behind our derivation of a model for describing the dynamics of a thin film on a flat substrate. First, by considering the conservation of mass of a thin liquid film on a horizontal substrate $\mathfrak{D} \subset \mathbb{R}^{n-1}$ where $n=2$ or 3 , we derive an equation for the height function of the film $h(\mathbf{x}, t)$ for $\mathbf{x} \in \mathfrak{D}, t \geq 0$.

We follow the standard argument of considering the horizontal flux $\mathbf{j}$ across an arbitrary sub-domain of the thin film, $\Omega \subset \mathfrak{D}$, such as presented in [8]. We consider the film to have constant density $\rho=1$, and that rate of mass lost across the interface of the film (due to evaporation for example) is given by $R$. From this, we obtain the conservation of mass equation

$$
\frac{\partial h}{\partial t}+\nabla \cdot \mathbf{j}=-R
$$

The constitutive classes of $\mathbf{j}$ and $R$ are chosen below.

The second key principle is a mechanical version of the second law of thermodynamics. This states that the increase in free energy of an arbitrary control volume $\Omega$ increases at a rate no greater than the work done on the region [7]. For some energy functional $\mathcal{F}(\Omega)=\int_{\Omega} \mathcal{E} d x$, this can be written as

$$
\frac{d}{d t} \mathcal{F}(\Omega)=\mathcal{W}(\Omega)-\mathcal{D}(\Omega)
$$


where $\mathcal{W}(\Omega)$ contains the work done on $\Omega$ and the free energy flux through the boundary $\partial \Omega$, and $\mathcal{D}(\Omega) \geq 0$ is the dissipation of the free energy.

The total energy density is given by $\mathcal{E}=\Psi+\Xi$, where $\Psi$ is the Helmholtz free energy density of the system, and $\Xi$ is a function encapsulating energies from other sources, including kinetic energy, energy from magnetic fields and thermal energy [16]. The constitutive class of $\Psi$, and the composition of $\Xi$ are a choice to be made by the modeler. In this work, we take $\Xi \equiv 0$, since non-zero $\Xi$ requires independent study.

\section{Constitutive Dependence}

Phase-field type models are driven by the variational derivative of the free energy functional. We follow arguments made in [5] and consider the Helmholtz free energy density $\Psi$ to depend on $h$ and its gradient, that is to say

$$
\Psi=\widehat{\Psi}(h, \nabla h),
$$

and the total energy functional is given by

$$
\mathcal{F}(\Omega)[h]:=\int_{\Omega} \widehat{\Psi}(h, \nabla h) d x .
$$

The variational derivative $\mu$ of $\mathcal{F}$ is defined as

$$
\mu=\frac{\delta \mathcal{F}}{\delta h}=\partial_{h} \widehat{\Psi}-\nabla \cdot\left(\partial_{\nabla h} \widehat{\Psi}\right) .
$$

An example of a classical choice for this energy which applies here is

$$
\widehat{\Psi}(h, \nabla h)=W(h)+\frac{\sigma^{2}}{2}|\nabla h|^{2},
$$

with corresponding variational derivative

$$
\mu=W^{\prime}(h)-\sigma^{2} \Delta h,
$$

where $W(h)$ is a free energy function depending only on the height $h$, and the second term is a surface energy contribution.

We now define a constitutive class for $\mathbf{j}$ and $R$ in equation (1) by postulating that these variables are dependent on $h$, the variational derivative $\mu$, and the gradients of these variables, that is to say

$$
\begin{aligned}
\mathbf{j} & =\widehat{\mathbf{j}}(h, \nabla h, \mu, \nabla \mu), \\
R & =\widehat{R}(h, \nabla h, \mu, \nabla \mu) .
\end{aligned}
$$

Having set up the constituent classes for the dependent variables in the model, we now derive constraints such that the second law of thermodynamics (2) holds. 


\section{Deriving Constraints}

We follow the procedure outlined in [5]. Using that $\Psi=\widehat{\Psi}(h, \nabla h)$ the left hand side of (2) equals:

$$
\frac{d}{d t}\left(\int_{\Omega} \widehat{\Psi}(h, \nabla h) d x\right)=\int_{\Omega}\left(\partial_{h} \widehat{\Psi} \partial_{t} h+\partial_{\nabla h} \widehat{\Psi} \cdot \partial_{t}(\nabla h)\right) d x,
$$

where $\partial_{x}$ is the partial derivative with respect to $x$. Switching the time and space derivatives in the last term of (10), integrating by parts, and using (5) we obtain

$$
\frac{d}{d t}\left(\int_{\Omega} \widehat{\Psi}(h, \nabla h) d x\right)=\int_{\Omega} \mu \partial_{t} h d x+\int_{\partial \Omega} \partial_{t} h \partial_{\nabla h} \widehat{\Psi} \cdot \mathbf{n} d s .
$$

We can now substitute (1) into (11), and integrating by parts the term involving $\mu \nabla \cdot \mathbf{j}$ gives

$$
\frac{d}{d t} \int_{\Omega} \Psi d x=-\int_{\Omega}(\mu R-\mathbf{j} \cdot \nabla \mu) d x+\int_{\partial \Omega}\left(-\mu \mathbf{j}+\partial_{t} h \partial_{\nabla h} \widehat{\Psi}\right) \cdot \mathbf{n} d s
$$

Comparing (12) to (2), we identify the domain integral to be the dissipation $\mathcal{D}(\Omega)$ and the boundary integral to be $\mathcal{W}(\Omega)$, which are natural identifications, similar as in earlier work [7].

Thus, a family of models that suitably describes the evolution of a thin film on a solid substrate while ensuring energy dissipation is given by

$$
\frac{\partial h}{\partial t}+\nabla \cdot \widehat{\mathbf{j}}=-\widehat{R}
$$

where $\widehat{\mathbf{j}}$ and $\widehat{R}$ are chosen to be as in (8) and (9) and

$$
\mu \widehat{R}-\widehat{\mathbf{j}} \cdot \nabla \mu \geq 0
$$

with $\mu=\partial_{h} \widehat{\Psi}-\nabla \cdot\left(\partial_{\nabla h} \widehat{\Psi}\right)$.

\section{Choices and Connections}

In this section, we show that the family of models described above is consistent with existing models for thin film evolution when the modeler makes particular choices for the constitutive relations.

Thiele's model [15] for a non-volatile case is given by

$$
\frac{\partial h}{\partial t}=\nabla \cdot\left[M_{c}(h) \nabla \frac{\delta \mathcal{F}}{\delta h}\right]
$$


where $M_{c}(h) \geq 0$ is the mobility function for the thin film and $\delta \mathcal{F} / \delta h$ is given in (5), with $\mathcal{F}$ given in (4). It is clear that this model fits into the framework (13) with $\widehat{R}(h, \nabla h, \mu, \nabla \mu)=0$ and $\widehat{\mathbf{j}}(h, \nabla h, \mu, \nabla \mu)=-M_{c}(h) \nabla \mu$, and with these choices it is also clear that constraint (14) is satisfied, implying the dissipation $\mathcal{D}(\Omega)=\int_{\Omega} M_{c}(h)|\nabla \mu|^{2} \geq 0$.

We now show that the model derived using asymptotic approaches by Burelbach et al [1] also satisfies these requirements. The equation for a non-volatile case $(R=0)$ given in [1] is

$$
\frac{\partial h}{\partial t}+S \nabla \cdot\left(h^{3} \nabla \Delta h\right)+\nabla \cdot\left(\left[A h^{-1}\right] \nabla h\right)=0,
$$

where $A \geq 0$ is a non-dimensionalised version of the Hamaker constant, $S \geq 0$ is the non-dimensionalised surface tension, and $\Delta=\nabla \cdot \nabla$.

Using the chain rule $\nabla f(h)=f^{\prime}(h) \nabla h$, with $f(h)=h^{-3}$, (16) can be rewritten as

$$
\frac{\partial h}{\partial t}+\nabla \cdot\left[-\frac{S h^{3}}{\sigma^{2}} \nabla\left(\frac{A \sigma^{2}}{3 S} h^{-3}-\sigma^{2} \Delta h\right)\right]=0 .
$$

By considering $\widehat{\Psi}(h, \nabla h)$ as in (6), and choosing

$$
W(h)=-\frac{A \sigma^{2}}{6 S} h^{-2}
$$

we observe that (17) can be rewritten in terms of the variational derivative $\mu$ given in (7), as

$$
\frac{\partial h}{\partial t}+\nabla \cdot\left[-\frac{S h^{3}}{\sigma^{2}} \nabla \mu\right]=0
$$

Hence, by choosing $\widehat{R}(h, \nabla h, \mu, \nabla \mu)=0$ and $\widehat{\mathbf{j}}(h, \nabla h, \mu, \nabla \mu)=-S h^{3} \sigma^{-2} \nabla \mu$ we see that the model fits the family defined in (13), with dissipation

$$
\mathcal{D}(\Omega)=\int_{\Omega} \frac{S h^{3}}{\sigma^{2}}|\nabla \mu|^{2} d x \geq 0
$$

for $h \geq 0$.

A point of interest here is that the so-called disjoining pressure $\Pi(h)$ chosen in the derivation of the model (16) is given by $\Pi(h)=-k W^{\prime}(h)=A h^{-3}$ for constant $k=\frac{3 S}{\sigma^{2}}$, and so is directly proportional to $-W^{\prime}(h)$, see also [14].

\section{Regularisation of the Asymptotic Model}

A characteristic of model (16) is that it breaks down as the film ruptures since $h^{-1} \rightarrow \infty$. In typical numerical simulations this breakdown is observed by $h$ 
becoming negative. To enable simulations to continue past the point of rupture one can regularise the bulk free energy $W(h)$ and the mobility function $m(h)=S h^{3} \sigma^{-2}$ as follows.

The dotted lines in Figure 1 show the non-regularised $m(h)$ and $W(h)$. To regularise the mobility, we force $m(h)=0$ for $h \leq 0$ (Figure 1, left). To handle $W(h)$, we choose a small $\epsilon>0$ and construct $W(h)$ to be quadratic for $h<\epsilon$, and remain as given in (18) for $h \geq \epsilon$. We require the minimum of $W(h)$ to be at $h=0$ and for the function to be continuous with a continuous derivative. The regularised function is given by

$$
W(h)= \begin{cases}\frac{1}{6 \epsilon^{4}} h^{2}-\frac{1}{3 \epsilon^{2}} & \text { if } h<\epsilon, \\ -\frac{1}{6} h^{-2} & \text { if } h \geq \epsilon .\end{cases}
$$

and is shown in Figure 1, right. This regularization leads to a potential $W(h)$ that is similar to those used in thin-film models with so-called pre-cursor films (although in our case the minimum of $W(h)$ is located at $h=0$ instead of the pre-cursor film thickness); see for more details, e.g., [13].
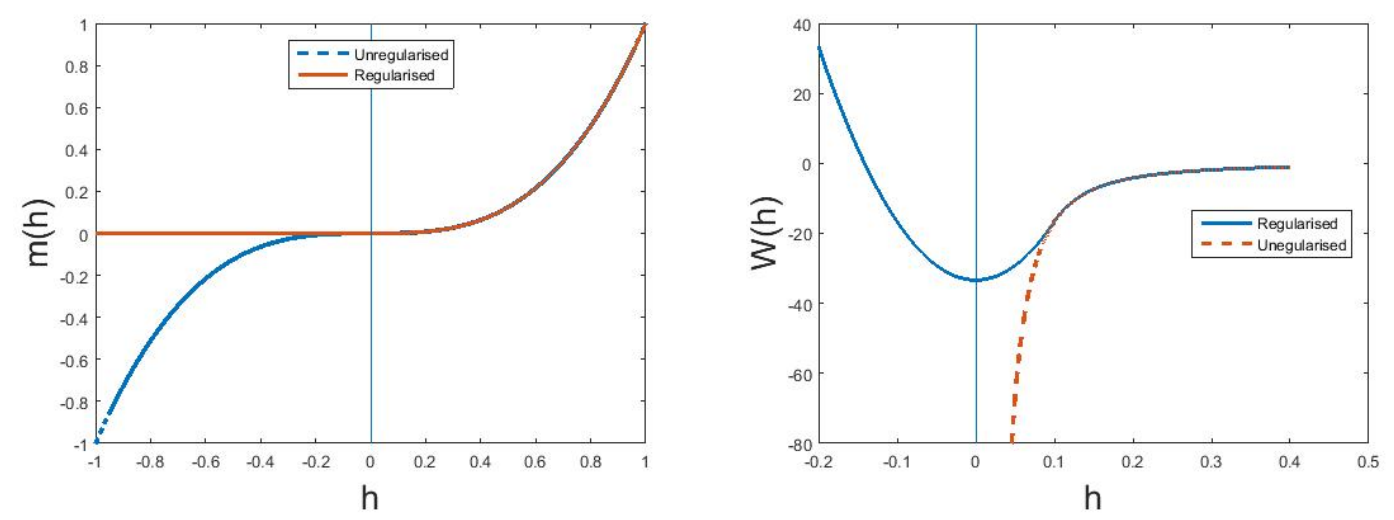

Figure 1: Graphs of the original mobility $m(h)$ (left) and free energy $W(h)$ (right), along with the regularised versions of these functions.

To perform numerical simulations we use a linear finite element discretisation in space for $h$ and $\mu$ in (19) and (7), employing homogeneous Neumann boundary conditions and triangular elements for the case of $n=3$. For the time discretisation we use a convex splitting method in which the non-linear term is split as $W(h)=$ $W_{+}(h)+W_{-}(h)$ with $W_{+}(h)$ being convex and $W_{-}(h)$ being concave. It is shown in [5] that if $W_{+}(h)$ is treated implicitly and $W_{-}(h)$ explicitly then the method is energy stable. In addition, if $\exists L_{W}>0$ such that $\left|W^{\prime \prime}(h)\right| \leq L_{W} \forall h$ then there exists a convex split with $W_{+}(h)=L_{W} h^{2} / 2$. This is a useful property as it results 
in the implicit terms being linear, removing the need to use a non-linear solver. Also, we use a semi-implicit treatment of the mobility term $m(h)$.
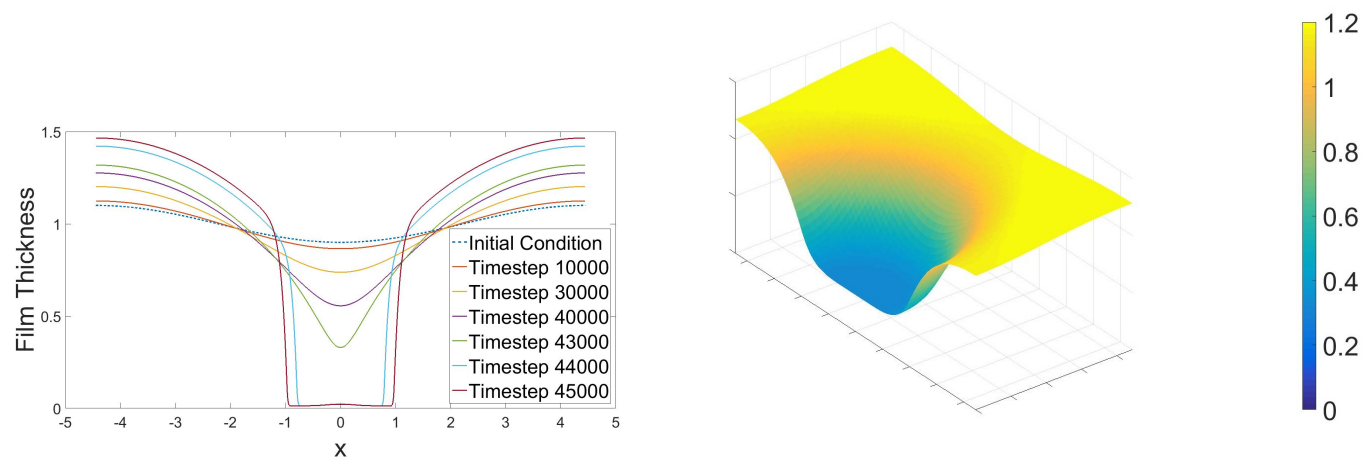

Figure 2: Left: Simulation with $n=2$ of the regularised asymptotic model showing film rupture in the domain $\mathfrak{D}=[-\pi \sqrt{2}, \pi \sqrt{2}]$. Right: Simulation with $n=3$ showing how a small perturbation in a flat thin film can result in a hole forming. Only half the domain $\mathfrak{D}=[-\pi \sqrt{2}, \pi \sqrt{2}] \times[0, \pi \sqrt{2}]$ is shown to visualise the dewetted area and the final time of $\mathrm{T}=6.25$.

Figure 2 shows examples of numerical solutions for $n=2$ (left) and $n=3$ (right). $\sigma, S$ and $A$ are taken to be 1 . For $n=2, \epsilon=0.1$ and $\Delta t=0.00032$, with an initial condition of $h(x, 0)=1-0.1 \cos (x / \sqrt{2})$. For $n=3, \epsilon=0.5, \Delta t=0.025$ and $h(x, y, 0)=1-0.05(\cos (x / \sqrt{2})+\cos (y / \sqrt{2}))$. The chosen initial conditions represent a small perturbation in a flat film.

It is clear that the small perturbation in the film grows until the film ruptures, at which point a hole forms and grows via dewetting.

\section{Conclusion}

In this work a family of thermomechanically consistent models for predicting the evolution of a non-volatile thin liquid film on a flat substrate was derived from mass conservation laws and the second law of thermodynamics, and it was shown that existing models fit within this family. In particular, this allows for regularisations that can be applied to modeling choices to better handle film rupture and dewetting.

In $[15,14,13]$ more complex thin-film processes are described that require a change in the energy functional $W(h)$, but the general form of the equation remains unchanged. Similarly, Lyushnin et al [10] postulate a different choice of $W(h)$ to simulate fingering instabilities. Further, it can be shown that other existing models, such as those developed in [4, 9] fit the framework, covering a 
wide range of applications from introducing a regime to account for slip to the growth of dry regions.

Current work being undertaken is directed at investigating volatile thin films, where $R \not \equiv 0$ in (1), as well as multiphase extensions, which can be used to simulate the evolution of a substance mixed with a volatile solvent, as in the fabrication of thin-film solar cells.

\section{Acknowledgements}

This work was funded by the Leverhulme Trust Modeling and Analytics for a Sustainable Society Grant. The contribution of the second author was partially supported by the Engineering and Physical Sciences Research Council (EPSRC) under grant EP/I036427/1.

\section{References}

[1] Burelbach, J. P., Bankoff, S.G., Davis, S.H.: Nonlinear stability of evaporating/condensing liquid films. J. Fluid Mech 195, 463-494 (1988)

[2] Coleman, B.D., Noll, W.: The thermodynamics of elastic materials with heat conductivity and viscosity. Arch. Ration. Mech. Anal. 13, 167-178 (1963)

[3] Eperon, G. E., Burlakov, V. M., Docampo, P., Goriely, A, Snaith, H. J.: Morphological control for high performance, solution-processed planar heterojunction Perovskite solar cells. Adv. Funct. Mater 24, 151-157 (2014)

[4] Fetzer, R., Jacobs, K., Munch, A., Wagner, B., Witelski, T. P.: New slip regimes and the shape of dewetting thin liquid films. Phys. Rev. Lett. (2005) doi: 10.1103/PhysRevLett.95.127801

[5] Gomez, H., van der Zee, K. G.:(2017) Computational phase-field modeling. In: Encyclopedia of Computational Mechanics, Second Edition. John Wiley \& Sons, Ltd.. ISBN 978-1-119-00379-3

[6] Gratzel, M.:The light and shade of perovskite solar cells . Nat. Mater. 13, 838-842 (2014)

[7] Gurtin, M. E.: Generalized Ginzburg-Landau and Cahn-Hilliard equations based on microforce balance. Phys. D. 92, 178-192 (1996)

[8] Gurtin, M. E., Fried, E., Anand, L.: The mechanics and thermodynamics of continua. Cambridge University Press (2010). 
[9] Kheshgi, H. S., Scriven, L. E.: Dewetting: Nucleation and growth of dry regions. Chem. Eng. Sci 46, 519-526 (1991)

[10] Lyushnin, A. V., Golovin, A. A., Pisman, L. M.: Fingering instability of thin evaporating liquid films. Phys. Rev. E. (2002) doi: 10.1103/PhysRevE.65.021602

[11] Oron, A., Davis, S. H., Bankoff, S. G.: Long-scale evolution of liquid thin films. Rev. Mod. Phys 69, 931-980 (1997)

[12] Pismen, L. M., Pomeau, Y.: Disjoining potential and spreading of thin liquid layers in the diffuse-interface model coupled to hydrodynamics. Phys. Rev. E. 62, 2480-2492 (2000)

[13] Sibley, D. N., Nold, A., Savva, N., Kalliadasis, S.: A comparison of slip, disjoining pressure, and interface formation models for contact line motion through asymptotic analysis of thin two-dimensional droplet spreading. J. Eng. Math. 94, 19-41 (2015)

[14] Thiele, U: Note on thin film equations for solutions and suspensions. Eur. Phys. J. Special Topics 197, 213-220 (2011)

[15] Thiele, U: Thin film evolution equations from (evaporating) dewetting liquid layers to epitaxial growth. J. Phys.:Condens.Matter 22, 1-11 (2010)

[16] Wodo, O, Ganapathysubramanian, B: Modeling morphology evolution during solvent-based fabrication of organic solar cells. Comput. Mater. Sci. 55, 113$126(2012)$ 